\title{
Comment on "Is a Trineutron Resonance Lower in Energy than a Tetraneutron Resonance?"
}

\author{
A. Deltuva* \\ Institute of Theoretical Physics and Astronomy, Vilnius University, Sauletekio al. 3, LT-10257 Vilnius, Lithuania \\ R. Lazauskas \\ IPHC, IN2P3-CNRS/Université Louis Pasteur BP 28, F-67037 Strasbourg Cedex 2, France
}

The quantum Monte Carlo study [1] of few-neutron resonant states provided results incompatible with rigorous few-body calculations [2 [4]. In this Comment we point out serious shortcomings in the framework of Ref. [1], leading to misinterpretation of unbound fewbody systems.

Study of unbound few-neutron systems [1] followed quite popular strategy consisting of two steps: (i) make the system bound with additional attractive potential, controlled by strength parameter $V_{0}$; (ii) extrapolate the resulting binding energy to the physical limit in continuum at $V_{0}=0$. Two different ways for step (i) have been employed in Ref. [1]: 1) adding an external trap potential and fixing center-of-mass (c.m) of the system; 2) enhancing the $n n$ interaction by factor $\alpha=1+V_{0}$. Such procedure is sound if (a) the calculated bound state is physical and it evolves into resonance, and (b) the analytic continuation to different Reemann sheet with resonance is performed correctly, taking into account threshold effects.

We argue that both these conditions are not satisfied in Ref. [1]. For definiteness we consider the fourneutron $(4 n)$ system. Additional attraction may generate a bound dineutron with energy $E_{d}<0$, which then defines the stability threshold for tetraneutrons: only those with $E_{4 n} \leq E_{d}$ in the trap (or those with $E_{4 n} \leq 2 E_{d}$ for the enhanced force) are stable. Otherwise, even in the case $E_{4 n}<0$ they can decay into dineutron plus two infinitesimally slow neutrons moving around the common mass center (trap) or into two dineutrons (produced by enhanced force).

Our study reveals that a bound dineutron emerges in trap with radius $R_{\mathrm{WS}}=6 \mathrm{fm}$ and potential depth $V_{0} \approx-0.09 \mathrm{MeV}$ only, or when the enhancement factor $\alpha$ in the ${ }^{1} S_{0}$ wave exceeds $\approx 1.1$ (these values slightly depend on the underlying $n n$ potential). However, $4 n$ states declared to be bound tetraneutrons with $E_{4 n} \rightarrow 0$ in Ref. 1] were found only at significantly larger absolute values of $V_{0} \approx-1.2 \mathrm{MeV}$ and $\alpha \approx 1.3$. For such Hamiltonians the dineutrons are already well bound, thus, the lowest-energy state of the system is not true (stable) bound state, but continuum state that asymptotically looks like dineutron in a trap plus two slow peripheral neutrons. It appears that Ref. [1] ignored this effect in the presumed $E_{4 n} \approx 0$ region, which is decisive for
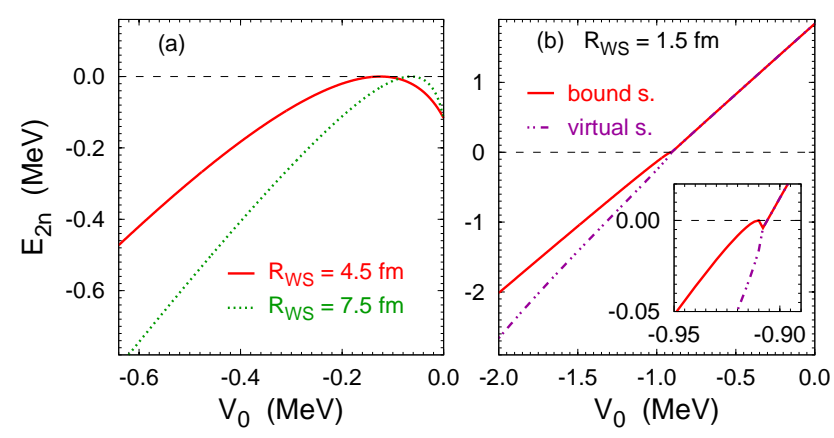

FIG. 1. ${ }^{1} S_{0}$ dineutron pole trajectories in Wood-Saxon traps with given range parameters for realistic (a) and two-Gaussian (b) potentials.

the extrapolation. The tetraneutron states of Ref. [1] are above the stability threshold and therefore are not true bound states but most probably represent some discretized continuum states that do not evolve into a resonance. Extrapolation of their energies does not lead to proper resonance energy.

Furthermore, a caution is needed in extrapolation procedure itself if real bound states are calculated, since trajectory of a bound state evolving into continuum state involves branching at each threshold with discontinuity in the second derivative of energy with respect to strength parameter [6]. Polynomial extrapolations [1] neglect this discontinuity and therefore are conceptually incorrect.

We show two examples in Fig. 1 corresponding to the ${ }^{1} S_{0}$ virtual state for a realistic potential and to the resonance of the two-Gaussian potential [1]. Obtained ${ }^{1} S_{0}$ pole trajectories have a typical bending shape, resulting $-0.12 \mathrm{MeV}$ virtual state energy, in sharp contrast with the positive $0.1 \mathrm{MeV}$ value of Ref. 1]. The latter is obtained by a polynomial extrapolation neglecting the near-threshold bending region. The resonance of the twoGaussian potential does not necessarily evolve from the ground state in the trap. In favorable case a linear extrapolation, avoiding the input from the near-threshold region, may give a reasonable estimation for the energy of a narrow resonance. However, presence of a branching point at the threshold, as shown in Fig. 11 (inset), produces highly nonlinear effects rendering naive extrapolation procedures mathematically unjustified. 
Authors acknowledge discussions with J. Carbonell. A.D. acknowledges the support by the Alexander von Humboldt Foundation under Grant No. LTU-1185721HFST-E.

* arnoldas.deltuva@tfai.vu.lt
[1] S. Gandolfi, H.-W. Hammer, P. Klos, J. E. Lynn, and A. Schwenk, Phys. Rev. Lett. 118, 232501 (2017).

[2] R. Lazauskas and J. Carbonell, Phys. Rev. C 72, 034003 (2005).

[3] E. Hiyama, R. Lazauskas, J. Carbonell, and M. Kamimura, Phys. Rev. C 93, 044004 (2016).

[4] A. Deltuva, Phys. Lett. B 782, 238 (2018).

[5] A. Deltuva, Phys. Rev. A 96, 022701 (2017).

[6] V.M. Krasnopolsky and V.I. Kukulin, Phys. Lett. A 69, 251 (1978). 